\title{
Successful treatment of postoperative, radiation-associated wound dehiscence around tracheostomy with negative pressure wound therapy.
}

Ewa Migacz, Dominik A. Walczak, Adam Maciejewski, Wojciech Kukwa

\section{CASE REPORT}

\begin{abstract}
We present a case report of a 62-year-old man who underwent a total laryngectomy, bilateral neck dissection and radiotherapy due to the laryngeal cancer. After 11 months he was reoperated because of a local recurrence. The second procedure was complicated with postoperative wound dehiscence in tracheostomy site which was successfully treated with negative pressure wound therapy. We indicate the difficulties and point technical solutions for negative pressure dressing around the airway.
\end{abstract}

Keywords-negative pressure wound therapy, tracheostomy, cervical wounds, head and neck wounds

\section{INTRODUCTION}

$\mathbf{N}$ EGATIVE pressure wound therapy (NPWT) has many features, which help in dealing with acute and chronic wound, i.e. maintaining a moist environment, optimizing blood flow, removing exudates, and applying pressure to promote wound closure ${ }^{1}$ However, scarce literature is available concerning application of NPWT in the head and neck region $\sqrt{2} 3$ The aim of this article is to describe a case report application of NPWT in a chronic wound management in a tracheostomy site, indicate the difficulties and suggest technical solutions for this type of wound healing management.

\section{CASE REPORT}

62-year-old patient following total laryngectomy, bilateral neck dissection and radiotherapy due to laryngeal cancer (01/2017) was scheduled for left neck dissection because of a local recurrence in 11/2017. The procedure was performed, and he was discharged 4 days later without complications. He was subsequently admitted to the emergency otolaryngology department 7 days after the surgery, because of the partial wound dehiscence in the scar adjacent to the tracheostomy (Fig. 11). A swab was taken from the wound, ciprofloxacin

Manuscript received 05.03.2018; revised 12.07.2018. This work did not receive any financial support. Authors declare no conflict of interest.

Author affiliations: Department of Otorhinolaryngology, Medical University of Warsaw, Warsaw, Poland, (EM, WK) ; Department of Oncological and Reconstructive Surgery, Maria Sklodowska-Curie Memorial Cancer Centre and Institute of Oncology, Gliwice, Poland, (DW, AM)

*Correspondence to: Ewa Migacz: ewamigacz@gmail.com

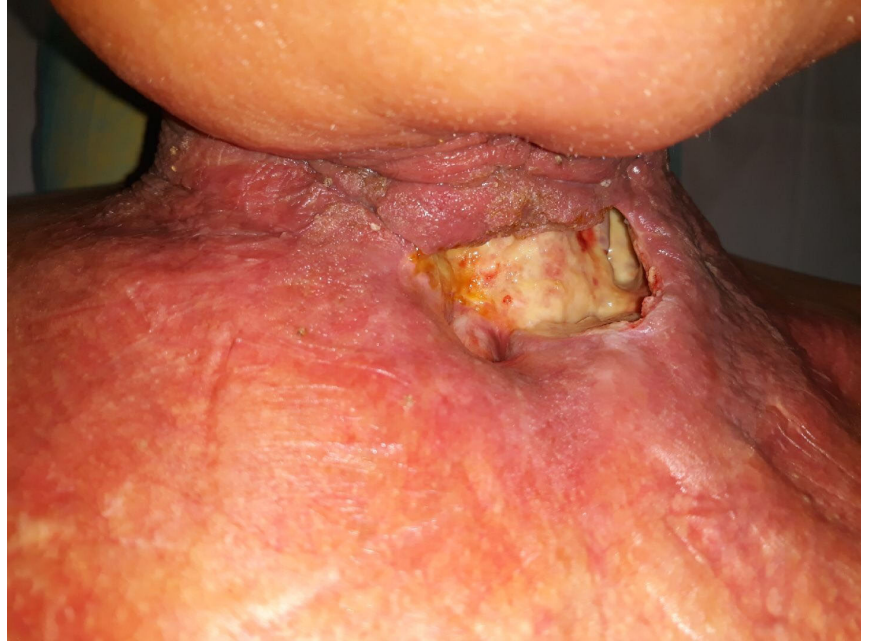

Figure 1. Wound before NPWT. Deep pouch is seen on the left side of the wound.

and clindamycin, were administered intravenously for Streptococcus constellatus and Pseudomonas aeruginosa, according to the results of antibiogram test. We applied conservative treatment for a week with no success, then we introduced NPWT. First we inserted cuffed tracheostomy tube (Portex) size 8.0 into the trachea to provide an airway. The balloon was inflated to create a seal between the tube and the trachea. Then the we applied polyurethane foam (RENASYS-F Foam, Smith \& Nephew) in a wound cavity around the tracheal tube. The stoma paste was placed over the tracheostomy tube to prevent air leak. Finally, we covered the wound with transparent dressing film(Fig. 2). The pump was set to a continuous negative pressure of $120 \mathrm{mmHg}$. The dressing was changed every 3rd day. The NPWT was well tolerated by the patient. We administered the negative pressure wound therapy for fifteen days. (Fig. 3 shows the result following the first treatment session.

When the wound got smaller, the adequate granulation tissue formation was achieved, and the epithelialization was noticed (Fig.4), the NPWT was stopped and advanced wound care dressings were applied (Intrasite Gel, Allevyn Ag NonAdhesive, Smith\&Nephew). 


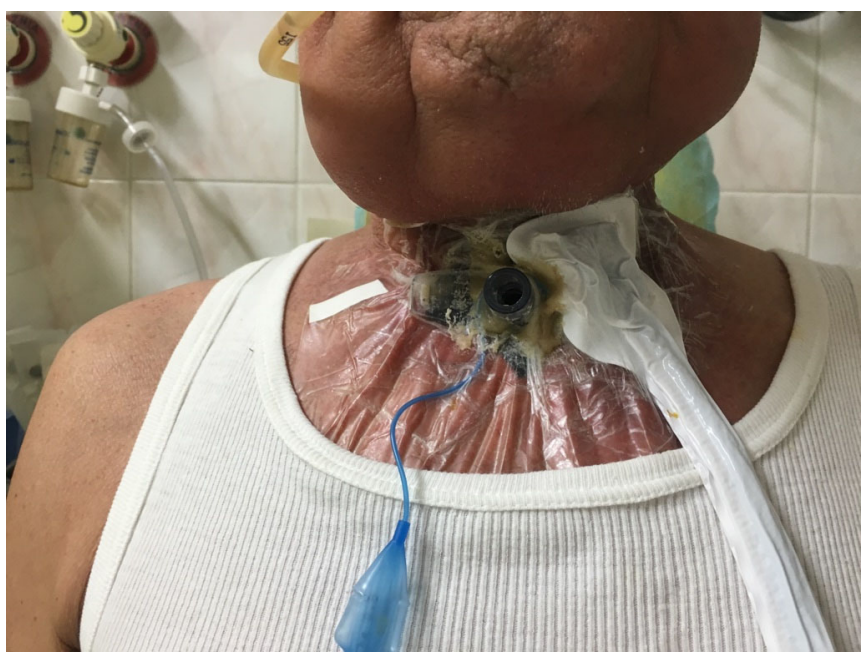

Figure 2. The application of NPWT. The stoma paste was used around the tracheostomy tube to tight the dressing.

\section{DISCUSSION}

Using negative pressure wound therapy as a treatment for open and infected wounds was first introduced by Wim Fleischmann et al. in 1993! This concept was later expanded by Argenta and Morykwas who developed the NPWT device in 1997! ${ }^{[5}$ Evolution of NPWT has become especially apparent in the last years and the range of indications for its use is still increasing. ${ }^{[6}$ Many publications have subsequently described the use of NPWT for the treatment of chronic and acute wounds in different locations, however there is still very few papers reporting the use of NPWT in the head and neck region. The NPWT exerts the number of beneficial clinical effects on a wound. It creates a moist environment, improves fluid removal, reduces tissue edema, contracts the wound, mechanically stimulates the wound bed, induces cell proliferation, alters blood flow in the wound edges, stimulates neo-angiogenesis and the formation of granulation tissue $]^{7} 8$

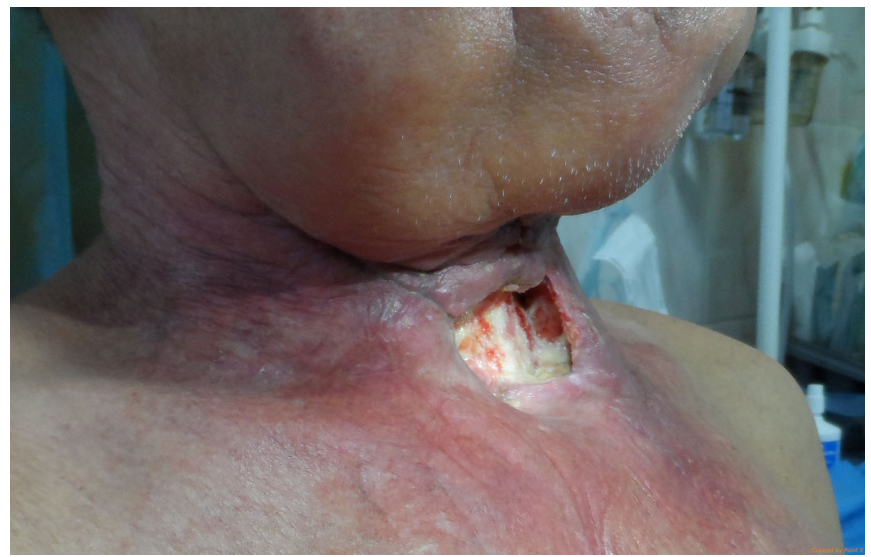

Figure 3. The wound after 3 days of NPWT.

The use of negative pressure wound therapy in neck region can be difficult to manage for a variety of reasons. First, many patients with complicated wounds in this region have a tracheostomy nearby, therefore, it is hard to achieve an

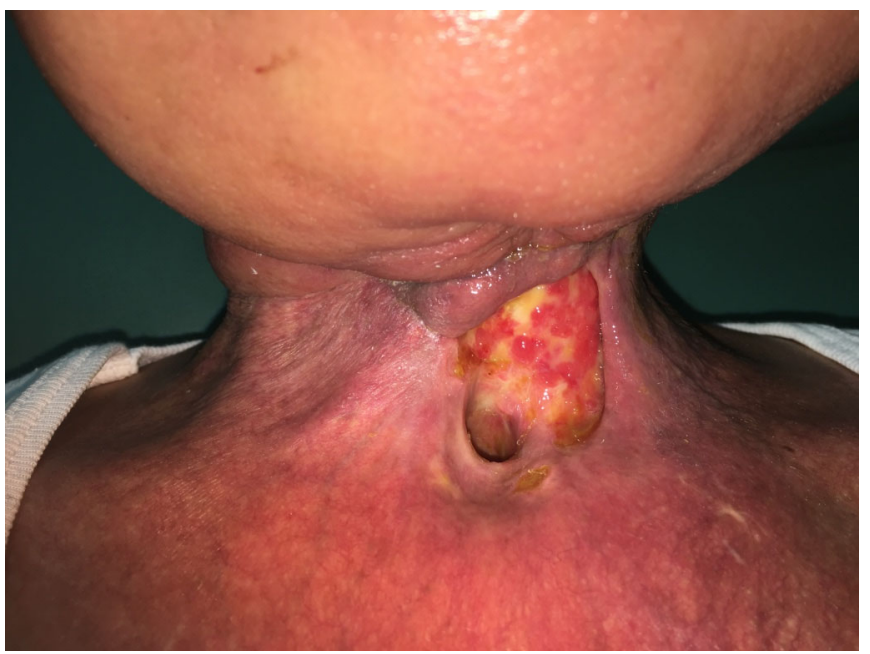

Figure 4. The result of 15 days of NPWT. Granulation tissue and epithelization is noticed. No pouch on the left side of wound is seen.

adequate air tight seal over and around the airway 9 Second, the presence of hair-bearing skin on the face and neck might also impair the seal of the dressing. Third, the neck surface is multi-faceted that is why placing a NPWT kit might be technically demanding. Fourth, using plastic tracheostomy tube with a cuff, to provide the sufficient seal of the dressing for few days requires the inhalations performed couple times per day to prevent from crusting of tube, nonetheless that may depressurize the dressing.

Additionally, many patients with wounds in the head and neck region have been treated with radiotherapy or radiochemotherapy, so the wound healing potential is compromised. There are only a few papers that describe negative pressure wound therapy as a safe and effective method in the treatment of radiation-associated wound complications. 10

The technique we have used to provide air seal of the NWPT dressing was based on a concept described by Johnston et al. However, instead of Aekin cohesive circular dressing we have used the stoma paste around the tracheostomy tube. The inflated cuff around the distal end of the cannula successfully prevented air leaks.

We used NPWT for 15 days and we achieved a reduction of the wound size, removal of the fibrin deposits, and formation of the granulation tissue. Moreover, the epithelialization was seen from the edges of the wound.

\section{CONCLUSIONS}

This case supports the previous observations that NPWT improves healing of complicated wounds in radiated area. Moreover, using tracheostomy tube and appropriate dressing a negative pressure wound therapy could be applied on wounds that develop around a laryngectomy stoma or tracheostomy site.

\section{REFERENCES}

[1] G. Han and R. Ceilley, "Chronic wound healing: a review of current management and treatments," Advances in therapy, vol. 34, no. 3, pp. 599-610, 2017. 
[2] S. Asher, H. White, J. Golden, J. Magnuson, W. Carroll, and E. Rosenthal, "Negative pressure wound therapy in head and neck surgery." JAMA facial plastic surgery., vol. 16, no. 2, pp. 120-6, 2014.

[3] M. Reiter and U. Harréus, "Vacuum assisted closure in the management of wound healing disorders in the head and neck: a retrospective analysis of 23 cases," American journal of otolaryngology, vol. 34, no. 5, pp. 411-415, 2013.

[4] W. Fleischmann, W. Strecker, M. Bombelli, and L. Kinzl, "Vacuum sealing as treatment of soft tissue damage in open fractures," Der Unfallchirurg, vol. 96, no. 9, pp. 488-492, 1993.

[5] L. Argenta and M. Morykwas, "Vacuum-assisted closure: a new method for wound control and treatment: clinical experience." 1997.

[6] L. Krokowicz, J. Borejsza-Wysocki, M Mackiewicz, and A. Iqbal, "10 years of negative pressure wound therapy (npwt)." Negative Pressure Wound Therapy., vol. 1, no. 1, pp. 22-32, 2014.

[7] N. Kairinos, A. M. Voogd, P. H. Botha, T. Kotze, D. Kahn, D. A. Hudson, and M. Solomons, "Negative-pressure wound therapy ii: negative-pressure wound therapy and increased perfusion. just an illusion?" Plastic and reconstructive surgery, vol. 123, no. 2, pp. 601$612,2009$.

[8] D. A. Walczak, M. Wojtyniak, R. Jaguścik, J. J. Porzeżyńska, W. Fałek,
M. Czerwińska, K. Ptasińska, L. Kozaczek, B. Szymański, and P. W. Trzeciak, "Management of large chronic venous leg ulcers with negative pressure wound therapy," Negative Pressure Wound Therapy Journal, vol. 4, no. 2, pp. 17-22, 2017.

[9] J. Johnston, F. Mariano, and D. Vokes, "Negative pressure dressing around the airway," The New Zealand medical journal, vol. 126, no. 1378, pp. 74-78, 2013.

[10] W. N. Welvaart, J. W. Oosterhuis, and M. A. Paul, "Negative pressure dressing for radiation-associated wound dehiscence after posterolateral thoracotomy," Interactive cardiovascular and thoracic surgery, vol. 8, no. 5, pp. 558-559, 2009.

[11] D.-m. Zhang, Z.-h. Yang, P.-1. Zhuang, Y.-y. Wang, W.-1. Chen, and B. Zhang, "Role of negative-pressure wound therapy in the management of submandibular fistula after reconstruction for osteoradionecrosis," Journal of Oral and Maxillofacial Surgery, vol. 74, no. 2, pp. 401-405, 2016.

[12] T. Nagata, M. Fujiwara, and H. Fukamizu, "Treatment of a radiation ulcer combining negative pressure wound therapy with flap reconstruction," The Journal of dermatology, vol. 40, no. 9, pp. 766-767, 2013. 